\title{
Categories whose objects are determined by their rings of endomorphisms
}

\section{Grigore Călugăreanu jr}

\begin{abstract}
In an additive category $A_{0}$, objects are said to be determined by their rings of endomorphisms if for each ring-isomorphism $F$ of the rings of endomorphisms of two objects $A, B$ in $A_{0}$ there is an isomorphism $f: A \rightarrow B$ in $A_{0}$ such that $F(\alpha)=f \alpha f^{-1}$, for every endomorphism $\alpha$ of $A$. Considering this problem in the context of closed categories (in Eilenberg and Kelly's sense), the author proves a general theorem which generalises results of Eidelheit (for real Banach spaces) and of Kasahara (for real locally convex spaces).
\end{abstract}

\section{Introduction}

Let $A, B$ be two objects in an additive category $A_{0}$. We consider the following problem: under what conditions on $A_{0}$ does a ring isomorphism $E: A_{0}(A, A) \rightarrow A_{0}(B, B)$ induce an isomorphism $f: A \rightarrow B$ in $A_{0}$, such that $F=A_{0}\left(f^{-1}, f\right)$, or equivalently, $F(\alpha)=f \alpha f^{-1}$, for every $\alpha \in A_{0}(A, A)$ ?

Examples of categories in which this problem has an affirmative answer are abundant. We concentrate on three of them, namely: the category of vector spaces and linear transformations over a division ring, the category

Received 23 March 1976. 
of real Banach spaces and linear continuous transformations (see [1]) and the category of real locally convex spaces and linear continuous transformations (see [3]).

Having these in mind, the notion of closed category (in the sense of [2]) is easily seen to be needed. According to this, we rephrase our initial problem in the following terms.

Let $\mathrm{F}:(A A) \rightarrow(B B)$ be an isomorphism in the closed category $A=\left(A_{0}, V\right.$, hom $\left.A, I, i, j, L\right)$, the subjacency of which is a ring homomorphism (and thus a ring-isomorphism). Under what conditions on $A$ is there an isomorphism $f: A+B$ in $A_{0}$ such that $F=\left(f^{-1}, f\right)$ ? We shall use the notations in the paper by Eilenberg and Kelly [2] and shall also denote by $E(A)$ the ring $A_{0}(A, A)$ of the endomorphisms of A .

\section{Preliminaries at the subjacent level}

From now on, let $A_{0}$ be an additive category with kernels and finite products (coproducts), and let $A, B$ be two objects in $A_{0}$.

LEMMA 1. If $P(A), P(B)$ denote the sets of direct factors (summands) of $A$ and $B$, respectively, then each ring-isomorphism $F: E(A) \rightarrow E(B)$ induces a canonical bijection $F^{*}: P(A) \rightarrow P(B)$.

Proof. By natural restriction, $F$ obviously induces a bijection between $E I(A)$ and $E I(B)$, the sets of idempotent endomorphisms of $A$ and $B$, respectively. It is then sufficient to indicate, for each object $A$ in $A_{0}$, a bijection $U_{A}: P(A) \rightarrow E I(A)$. If $A_{1}$ is a direct factor of $A$, and $p_{1}, u_{1}$ are the canonical projection and injection, respectively, then defining $U_{A}\left(A_{1}\right)=u_{1} p_{1}$ and $U_{A}^{-1}(\theta)=\operatorname{ker}(1-\theta)$ for every $\theta \in E I(A), U_{A}$ and $U_{A}^{-1}$ are easily seen to be mutually inverse (see [4], $I, 18.5)$. So $F^{*}=U_{B}^{-1} \cdot F / E I(A) \cdot U_{A}$.

REMARK. Considering $P(A)$ preordered by the well-known relation of comparing subobjects, one can easily verify that $F^{*}$ is actually a 
preorder isomorphism. Indeed, one has to show that if $\left(A_{1}, u_{1}\right) \leq\left(A_{2}, u_{2}\right)$ then $\left(F^{*}\left(A_{1}\right), u_{1}^{*}\right) \leq\left(F^{*}\left(A_{2}\right), u_{2}^{*}\right)$; but this follows immediately from $\left(1_{B}-F\left(u_{2} p_{2}\right)\right) \cdot u_{1}^{*}=0$.

COROLLARY. Under the assumptions of the previous Zemma, if $A=\prod_{i=1}^{n} A_{i}$ then $B=\prod_{i=1}^{n} F^{*}\left(A_{i}\right)$.

Proof. Let $\left(u_{i}: A_{i}+A\right)_{i=1}^{n}$ and $\left(p_{i}: A \rightarrow A_{i}\right)_{i=1}^{n}$ be the injections and projections of the biproduct $A$. We then have $p_{i j} u_{j}=\delta_{i j}$ and $\sum_{i=1}^{n} u_{i} p_{i}=I_{A}$. The morphisms $u_{i} p_{i}$ being idempotent, $F\left(u_{i} p_{i}\right)$ have the same property, and we have $F^{*}\left(A_{i}\right)=\operatorname{ker}\left(1_{B}-F\left(u_{i} p_{i}\right)\right)$. From $\left(I_{B}-F\left(u_{i} p_{i}\right)\right) \cdot F\left(u_{i} p_{i}\right)=0$ follows the unique existence of morphisms $p_{i}^{*}$ such that $F\left(u_{i} p_{i}\right)=u_{i}^{*} \cdot p_{i}^{*}, u_{i}^{*}$ denoting the infection of the direct factor $F^{*}\left(A_{i}\right)$ in $B$.

According to $([4], I, 18.1)$ we only have to show that $\sum_{i=1}^{n} u_{i}^{*} p_{i}^{*}=1_{B}$ and $p_{i}^{*} \cdot u_{j}^{*}=\delta_{i j}$. The first equality is obvious because

$$
\sum_{i=1}^{n} u_{i}^{*} \cdot p_{i}^{*}=\sum_{i=1}^{n} F\left(u_{i} p_{i}\right)=F\left(\sum_{i=1}^{n} u_{i} p_{i}\right)=F\left(1_{A}\right)=1_{B},
$$

any ring-isomorphism being unital. As for the second, from $u_{i}^{*}=\operatorname{ker}\left(I_{B}-F\left(u_{i} p_{i}\right)\right)=\operatorname{equ}\left(I_{B}, F\left(u_{i} p_{i}\right)\right)$ we derive

$$
u_{i}^{*}=F\left(u_{i} p_{i}\right) \cdot u_{i}^{*}=u_{i}^{*} \cdot p_{i}^{*} \cdot u_{i}^{*}
$$

and $u_{i}^{*}$ being mono, we have $p_{i}^{*} \cdot u_{i}^{*}=1$. For $i \neq j$ we also have $u_{i} \cdot p_{i} \cdot u_{j} \cdot p_{j}=0$, and then $F\left(u_{i} p_{i}\right) \cdot F\left(u_{j} p_{j}\right)=u_{i}^{*} \cdot p_{i}^{*} \cdot u_{j}^{*} \cdot p_{j}^{*}=0$. So $p_{i}^{*} \cdot u_{j}^{*}=0$ follows, $p_{j}^{*}$ being epi (in fact, a retraction).

REMARK. Applying this corollary to $F^{-1}$ and $\left(F^{*}\right)^{-1}$, one verifies that $A^{\prime}$ is an indecomposable factor of $A$ iff $F^{\star}\left(A^{\prime}\right)$ is an 
indecomposable factor of $B$.

LEMMA 2. If $\left(A_{1} ; u_{1}, p_{1}\right)$ is a direct factor of $A$ then there is a ring-isomorphism $E\left(A_{1}\right) \rightarrow\left(u_{1} p_{1}\right) E(A)\left(u_{1} p_{1}\right)$.

Proof. If we define $W_{1}: E\left(A_{1}\right) \rightarrow\left(u_{1} p_{1}\right) E(A)\left(u_{1} p_{1}\right)$ and

$W_{1}^{-1}:\left(u_{1} p_{1}\right) E(A)\left(u_{1} p_{1}\right) \rightarrow E\left(A_{1}\right)$ by $W_{1}\left(\theta_{1}\right)=u_{1} p_{1} \theta_{1} u_{1} p_{1}$ and

$W_{1}^{-1}\left(u_{1} p_{1} \theta u_{1} p_{1}\right)=p_{1} \theta u_{1}$, respectively; these are easily seen to be mutually inverse ring-homomorphisms.

COROLLARY. Under the assumptions of the previous lemma we have a ring-isomorphism $E\left(A_{1}\right) \rightarrow E\left(F^{*}\left(A_{1}\right)\right)$.

Proof. We have only to notice that if $E(A)$ and $E(B)$ are ringisomorphic then $u_{1} p_{1} E(A) u_{1} p_{1}$ and $u_{1}^{*} \cdot p_{1}^{*} E(B) u_{1}^{*} \cdot p_{1}^{*}$ are also ringisomorphic.

We are now in a position to prove the main subjacent-level result:

THEOREM 1. Let $A_{0}$ be an additive category with kernels and finite products (coproducts), Let $A, B$ be objects in $A_{0}$, and let $U$ be $a$ direct factor of $A$. If $F: E(A) \rightarrow E(B)$ is a ring-isomorphism then there is a semi-Zinear isomorphism of abelian groups $E_{U}: A_{0}(U, A) \rightarrow A_{0}\left(F^{*}(U), B\right)$, that is to say, $E_{U}$ is a group homomorphism and $F_{U}(\alpha \theta)=F(\alpha) \cdot F_{U}(\theta)$ holds for each $\alpha$ in $E(A)$ and $\theta$ in $A_{0}(U, A)$.

Proof. Let $u$ and $p$, respectively, be the injection and the projection of $U$ in $A$. Define $F_{U}(\theta)=F(\theta p) \cdot u^{*}$, for each $\theta \in A_{0}(U, A)$, where $u^{*}, p^{*}$ denote the injection and the projection of $F^{*}(U)$ in $B$, respectively. It is only routine to verify that $F_{U}$ is a group-homomorphism which is semi-linear (in the sense described above). $F_{U}^{-1}: A_{0}\left(F^{*}(U), B\right) \rightarrow A_{0}(U, A)$, defined by $F_{U}^{-1}\left(\theta^{*}\right)=F^{-1}\left(\theta^{*} p^{*}\right) \cdot u$, is easily checked to be a two-sided inverse for $F_{U}$. 


\section{The main theorem}

Let $A=\left(A_{0}, V\right.$, hom $\left.A, I, i, j, L\right)$ be a closed category. We shall be concerned with the following conditions:

Al: $A_{0}$ is an additive category with kernels and finite (bi)products;

A2: $\quad V$ is a faithful functor;

A3: for each nonzero object $A$ in $A_{0}, A_{0}(I, A)$ contains a coretraction;

A4I: the object $I$ is indecomposable (into direct (bi)products);

A42: according to $A 3$, considering, $I$ as a direct factor of $A$, for each ring-isomorphism $F: E(A) \rightarrow E(B)$ there exists an isomorphism $w_{I}: I \rightarrow F^{*}(I)$.

THEOREM 2. Let A be a closed category which satisfies the conditions $\mathrm{A} 1, \mathrm{~A} 2, \mathrm{~A} 3$, and one of the conditions $\mathrm{A} 41, \mathrm{~A} 42$. If for two objects $A, B$ in $A_{0}$ there is an isomorphism $F:(A A) \rightarrow(B B)$ in $A_{0}$, the subjacency of which is a ring-homomorphism, then there is a canonical isomorphism $f: A \rightarrow B$; that is $F=\left(f, f^{-1}\right)$.

Proof. According to $A 3$ we shall denote by $p_{A}$ and $u_{A}$, respectively, the projection and the injection of $I$ in $A$, and by $p_{B}^{*}, u_{B}^{*}$ the projection and the injection of $F^{*}(I)$ in $B$, respectively.

First, let us show that the morphism $\mathrm{F}_{I}:(I A) \rightarrow\left(F^{*}(I), B\right)$ in $\mathrm{A}_{0}$ given by $F_{I}=\left(u_{B}^{*}, l_{B}\right) \cdot F \cdot\left(p_{A}, l_{A}\right)$ is an isomorphism. We note that if $F=V(F)$, then $F_{I}=V\left(F_{I}\right)$. We shall prove that

$\mathrm{F}_{I}^{-1}:\left(F^{*}(I), B\right) \rightarrow(I A)$, given by $\mathrm{F}_{I}^{-1}=\left(u_{A}, I_{A}\right) \cdot \mathrm{F}^{-1} \cdot\left(p_{B}^{*}, I_{B}\right)$, is a twosided inverse for $F_{I}$. In order to prove that

$$
\begin{aligned}
& \mathrm{F}_{I}^{-1} \cdot \mathrm{F}_{I}=\left(u_{A}, 1_{A}\right) \cdot \mathrm{F}^{-1} \cdot\left(u_{B}^{*} p_{B}^{*}, 1_{B}\right) \cdot \mathrm{F} \cdot\left(p_{A}, 1_{A}\right), \\
& \mathrm{F}_{I} \cdot \mathrm{F}_{I}^{-1}=\left(u_{B}^{*}, 1_{B}\right) \cdot \mathrm{F} \cdot\left(u_{A} p_{A}, 1_{A}\right) \cdot \mathrm{F}^{-1} \cdot\left(p_{B}^{*}, 1_{B}\right)
\end{aligned}
$$


are both identities, it is sufficient to prove that

$$
\left(u_{B}^{*} p_{B}^{*}, l_{B}\right) \cdot \mathrm{F}=\mathrm{F} \cdot\left(u_{A} p_{A}, \mathrm{I}_{A}\right),
$$

because $p_{A} u_{A}=1, p_{B}^{*} u_{B}^{*}=1$. The subjacency functor being faithful, it is sufficient to check this equality at the subjacent level, namely,

$$
A_{0}\left(u_{B}^{*} p_{B}^{*}, I_{B}\right) \cdot F=F \cdot A_{0}\left(u_{A} p_{A}, I_{A}\right) \text {. }
$$

Applying both members to a $\theta \in E(A)$, one has $u_{B}^{*} p_{B}^{*}=F\left(u_{A} p_{A}\right)$, which is true (see the proof of the corollary of the first lemma).

Now, using the remark following the same corollary, from A4I, I being indecomposable, $F^{*}(I)$ is also indecomposable and so, again by $A 3$, there is an isomorphism $w_{I}: I \rightarrow F^{*}(I)$. If we choose the condition $A 42$ instead of $\mathrm{A} 41$, such an isomorphism $w_{I}$ also exists, by hypothesis.

We are now in a position to define the canonical isomorphism $f: A \rightarrow B$ as follows: $f=i_{B}^{-1} \cdot\left(w_{I}, 1_{B}\right) \cdot F_{I} \cdot i_{A}$, where $i$ is the natural isomorphism given with the closed category structure in $A$. It is clear that $f$, as composite of isomorphisms, is also an isomorphism.

The functor $V$ being faithful, the two ways of requiring the canonicity of $f$, namely, $V(F)(\alpha)=f \cdot \alpha \cdot f^{-1}$ and $F=\left(f^{-1}, f\right)$, are equivalent. We adopt the first one, which is also equivalent to $F(\alpha) \cdot f=f . \alpha$ for each $\alpha \in E(A)$.

One has to verify that

$$
\begin{aligned}
F(\alpha) \cdot i_{B}^{-1} \cdot\left(w_{I}, 1_{B}\right) \cdot\left(u_{B}^{*}, 1_{B}\right) \cdot \mathrm{F} \cdot\left(p_{A}, 1_{A}\right) \cdot i_{A}= \\
\quad=i_{B}^{-1} \cdot\left(w_{I}, 1_{B}\right) \cdot\left(u_{B}^{*}, 1_{B}\right) \cdot \mathrm{F} \cdot\left(p_{A}, 1_{A}\right) \cdot i_{A} \cdot \alpha .
\end{aligned}
$$

First, we note that

$$
i_{A} \cdot \alpha \cdot i_{A}^{-1}=\left(1_{I}, \alpha\right)
$$

and

$$
i_{B} \cdot F(\alpha) \cdot i_{B}^{-1}=\left(1_{I}, F(\alpha)\right)
$$


are true, because of the naturality of the isomorphism $i$. Thus it only remains to show that

$$
\left(u_{B}^{*} \cdot w_{I}, F(\alpha)\right) \cdot \mathrm{F} \cdot\left(p_{A}, 1_{A}\right)=\left(u_{B}^{*} \cdot w_{I}, I_{B}\right) \cdot \mathrm{F} \cdot\left(p_{A}, \alpha\right) .
$$

But this can be readily checked at the subjactnt level: the equality $F(\alpha) \cdot F\left(\alpha \cdot p_{A}\right) \cdot u_{B}^{*} \cdot w_{I}=F\left(\alpha \cdot \theta \cdot p_{A}\right) \cdot u_{B}^{*} \cdot w_{I}$ is true for every $\theta \in A_{0}(I, A), \quad F$ being a ring homomorphism. This completes our proof.

\section{Several applications and comments}

Let us denote by $K$ the category of vector spaces and linear transformations over a division ring $K$, by $B$ the category of real Banach spaces and linear continuous transformations, and by $L$ the category of real locally convex spaces and linear continuous transformations.

First, it is obvious that $A$ is fulfilled in any one of the three categories considered above. $K$ and $B$ have well-known structures of closed categories, and $L$ also admits such a structure, obtained by considering on the vector space of the linear continuous transformations the locally convex topology of $\sigma$-convergence, with $\sigma$ the family of the bounded subsets of the domain.

Next, for these closed categories, the division ring $K$ in $K$ and the real line in $B$ and $L$ are the corresponding objects $I$. Indecomposables are one-dimensional spaces and so $A 41$ is fulfilled. All these categories being concrete, the Condition A2 is satisfied. Finally, Condition A3 holds in all of these categories, because for each nonzero element in a space in any of these categories, there is a nontrivial functional which takes the value 1 (identity of $K$ or real number, respectively) on this element.

In fact, the largest category of topological vector spaces in which Condition $\mathrm{A} 3$ is satisfied contains all the spaces which admit a nontrivial functional, or equivalently (by a theorem of LaSalle), those which contain a proper open and convex subset. Unfortunately this is not a "nice" category for the rest of our conditions.

From another point of view, let $R$ be an associative ring with identity, and let $R$-mod be the closed category of left unitary 
$R$-modules. One can now raise the problem: $A 1, A 2$ being satisfied in $R$-mod, under what conditions on the ring $R$ are Condition $A 3$ and one of the Conditions $\mathrm{A} 41$ and $\mathrm{A} 42$ satisfied?

Unfortunately again, the answer is a deceiving one: $R$ must be a division ring. Indeed, Condition A3 implies the following concrete condition: the left $R$-module $R^{R}$ must be a direct summand of every nonzero left $R$-module.

Let us suppose that for a ring $R$ this condition holds. Let $R^{M}$ be a simple left $R$-module (such ones do exist, for example, $R / m$ for a left maximal ideal $m$ in $R$, considered as a left $R$-module). $R^{R}$ being isomorphic with a cyclic submodule of $R^{M}$, it follows that $R^{R}$ is actually isomorphic with $R^{M}$, and so simple as a left $R$-module.

Then $R$ is a not necessarily commutative division ring. For this last comment, I am indebted to Mr Nae Popescu.

\section{References}

[1] M. Eidelheit, "On isomorphisms of rings of linear operators", Studia Math. 9 (1940), 97-105.

[2] Samuel Eilenberg and G. Max Kelly, "Closed categories", Proceedings of the Conference on Categorical Algebra, La Jolla, 1965, 421-562 (Springer-Verlag, Berlin, Heidelberg, New York, 1966).

[3] Shouro Kasahara, "Sur l'espace des endomorphismes continus de l'espace vectoriel localement convexe", Math. Japon. 3 (1955), 111-116.

[4] Barry Mitchell, Theory of categories (Pure and Applied Mathematics, 17. Academic Press, New York and London, 1965).

Department of Mathematics, Universitatea "Babes-Bolyai", Cluj-Napoca, Roumania. 